\title{
ON ALGEBRAIC SIMPLE MONIC SETS OF POLYNOMIALS
}

\section{RAGY AND BUSHRA H. MAKAR}

1. [7]. ${ }^{1}$ A set of polynomials $\left\{p_{n}(z)\right\} \equiv p_{0}(z), p_{1}(z), p_{2}(z), \cdots$ is said to be simple if for all $n, p_{n}(z)$ is of degree $n$. We call the set monic if for all $n$ the coefficient of $z^{n}$ in $p_{n}(z)$ is unity. Such a set is basic, that is, every polynomial can be expressed uniquely as a finite linear combination of the polynomials $p_{0}(z), p_{1}(z), p_{2}(z), \cdots$. In particular,

$$
z^{n}=\sum_{i=0}^{n} \pi_{n i} p_{i}(z), \quad \pi_{n n}=1,
$$

$\Pi=\left[\pi_{i j}\right]$ being the reciprocal matrix of $P=\left[p_{i j}\right]$, where

$$
p_{i}(z)=\sum_{j=0}^{i} p_{i j} z^{j}, \quad p_{i i}=1 .
$$

Each of $P$ and $\Pi$ is a lower-semi matrix with all elements in the leading diagonal unity.

Given an integral function $f(z)=\sum_{n=0}^{\infty} a_{n} z^{n}$, there is an associated series

$$
\Pi_{0} p_{0}(z)+\Pi_{1} p_{1}(z)+\Pi_{2} p_{2}(z)+\cdots
$$

where

$$
\Pi_{n}=a_{n}+a_{n+1} \pi_{n+1, n}+a_{n+2} \pi_{n+2, n}+\cdots .
$$

The set is said to represent $f(z)$ if the associated series converges uniformly to $f(z)$ in every finite part of the plane.

The expression

$$
\omega=\lim _{R \rightarrow \infty}\left\{\limsup _{n \rightarrow \infty} \frac{\log \omega_{n}(R)}{n \log n}\right\}
$$

where

$$
\omega_{n}(R)=\sum_{i=0}^{n}\left|\pi_{n i}\right| A_{i}(R), \quad A_{i}(R)=\max _{|z|=R}\left|p_{i}(z)\right|,
$$

is called the order of the set and is of essential importance in that a set of order $\omega$ represents every integral function of order less than $1 / \omega$.

Received by the editors June 5, 1950.

1 Numbers in brackets refer to the references at the end of the paper. 
2. An infinite matrix $A$ is said to be algebraic [1] if it is self-associative and satisfies an equation of the form

$$
\alpha_{0} A^{m}+\alpha_{1} A^{m-1}+\alpha_{2} A^{m-2}+\cdots+\alpha_{m} I=0,
$$

where $I$ is the infinite unit matrix. If this equation is the equation of least degree satisfied by the matrix $A, A$ is said to be algebraic of degree $m .^{2}$

Such an equation of least degree may be looked upon from some point of view as to correspond to the reduced [5] characteristic equation of a square matrix, but with the clear understanding that every square matrix has a reduced characteristic equation, which is sometimes the characteristic equation itself, while only algebraic infinite matrices satisfy such algebraic equations.

A lower-semi matrix is self-associative, and so is algebraic if it merely satisfies an algebraic equation. The characteristic equation of a square matrix of order $n \times n$ in which all elements in the leading diagonal are unity and all elements above the leading diagonal are zero is $(A-I)^{n}=0$, and hence the reduced characteristic equation of such a matrix is necessarily $(A-I)^{k}=0, k \leqq n$. By mere induction, it follows that a lower-semi matrix $P$ in which all elements in the leading diagonal are unity, if algebraic of degree $m$, must satisfy the equation $(P-I)^{m}=0$, which is

$$
P^{m}-m_{c_{1}} P^{m-1}+m_{c_{2}} P^{m-2}-\cdots+(-1)^{m} I=0 .
$$

The simple monic set of polynomials $\left\{p_{n}(z)\right\}$ whose matrix of coefficients is $P$ may be called an algebraic set of degree $m$, and we have: $\left\{p_{n}(z)\right\}^{m}-m_{c_{1}}\left\{p_{n}(z)\right\}^{m-1}+\cdots+(-1)^{m}\left\{z^{n}\right\} \equiv 0$.

3. The order of a simple monic set whose coefficients are of certain order of magnitude has been investigated. Thus it has been shown that if in such a set $\left|p_{n i}\right| \leqq k n^{\lambda}, n=1,2,3, \cdots, i=0,1,2, \cdots, n-1$, then the set is of order at most $\lambda[6]$, and a set has been constructed whose order is the upper bound $\lambda$. The same upper bound has been obtained [2] for the order of a simple monic set in which $\left|p_{n i}\right|$ $\leqq k n^{\lambda(n-i)}$.

It is worthy of remark that $\lambda$ is not the upper bound of the order of a simple monic set in which $\left|p_{n i}\right| \leqq k n^{\lambda n}, n=1,2,3, \cdots ; i=0$, $1,2, \cdots, n-1$. An obvious example is the set $\left\{p_{n}(z)\right\}$ defined by

$$
p_{0}(z)=1, \quad p_{n}(z)=-n^{\lambda n} z^{n-1}+z^{n}, \quad n \geqq 1,
$$

2 We rather prefer the term "degree" than the original term "order" given in $[1]$, since the term "order" is necessarily used here with a different meaning. 
which is of infinite order. The reduced characteristic equation of the square matrix

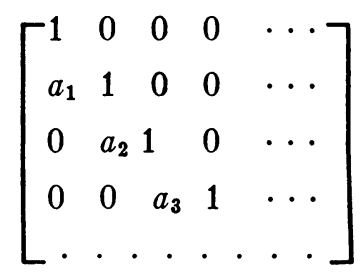

of order $n \times n, a_{1}, a_{2}, \cdots, a_{n-1} \neq 0$, is easily seen to be $(A-I)^{n}=0$, so that making $n \rightarrow \infty$, the matrix of coefficients $P$ of the above set does not satisfy an algebraic equation. In other words, the set $\left\{p_{n}(z)\right\}$ is not an algebraic set.

We show that the order of an algebraic simple monic set in which $\left|p_{n i}\right| \leqq k n^{\lambda n}$ has an upper bound which is finite, and may be attained.

In the case of a simple monic set in which $\left|p_{n i}\right| \leqq k n^{\lambda(n-i)}, n$ $=1,2,3, \cdots ; i=0,1,2, \cdots, n-1$, the set $\left\{p_{n}(z)\right\}$ and its powers $\left\{p_{n}(z)\right\}^{\nu}$ are all [2] of order $\lambda$ at most. The factor $n^{-\lambda i}$ plays an important part. In the case of an algebraic simple monic set of degree $m$ satisfying (3.1), such a factor is not present and a similar result is not expected. It may be noticed at once that the coefficients of the power set $\left\{p_{n}(z)\right\}^{\nu}$ may become of larger and larger order of magnitude as $\nu$ increases until $\nu$ reaches the value $m-1$, when the algebraic equation (2.1) seems to stop such increase in the order of magnitude of coefficients, for the set $\left\{p_{n}(z)\right\}^{m}$ and higher powers. We may therefore expect the order of $\left\{p_{n}(z)\right\} \nu$ to increase with $\nu$, until $\nu$ reaches the value $m-1$, when the order becomes fixed. A complete investigation of the growth of such an order is given.

THEOREM 1. If $\left\{p_{n}(z)\right\}$ is an algebraic simple monic set of degree $m$ in which

$$
\left|p_{n i}\right| \leqq k n^{\lambda n}, \quad k \geqq 1 ; n=1,2,3, \cdots ; i=0,1,2, \cdots, n-1,
$$

then $\left\{p_{n}(z)\right\}^{\nu}$ is of order at most $(m+\nu-1) \lambda, 1 \leqq \nu \leqq m-1,\left\{p_{n}(z)\right\}$ " is of order at most $2(m-1) \lambda, \nu \geqq m-1$. The upper bound may be attained in all cases.

Equation (2.1) is

$$
I=m_{c_{1}} P-m_{c_{2}} P^{2}+m_{c_{3}} P^{3}-\cdots+(-1)^{m-1} P^{m} .
$$

Multiplying by $\Pi=P^{-1}$ we get

$$
\Pi=m_{c_{1}} I-m_{c_{2}} P+m_{c_{2}} P^{2}-\cdots+(-1)^{m-1} P^{m-1} .
$$


If we write $P^{v}=p_{i j}^{(v)}$, then

$$
p_{n i}^{(2)}=p_{n i}+\sum_{j=i+1}^{n-1} p_{n j} p_{j i}+p_{n i}
$$

so that by (3.1)

$$
\left|p_{n i}^{(2)}\right| \leqq k^{2} n^{\lambda n} \sum_{j=i+1}^{n-1} j^{\lambda j}+2 k n^{\lambda n}
$$

that is,

(3.4) $\left|p_{n i}^{(2)}\right|<k^{2}(n+1) n^{2 \lambda n}, \quad n=1,2,3, \cdots ; i=0,1,2, \cdots, n$.

Also

$$
p_{n i}^{(3)}=p_{n i}^{(2)}+\sum_{j=i+1}^{n-1} p_{n j}^{(2)} p_{j i}+p_{n i}
$$

so that by (3.1) and (3.4)

$$
\left|p_{n i}^{(3)}\right|<k^{3}(n+1)^{2} n^{3 \lambda n}
$$

In general,

$$
\begin{aligned}
& \left|p_{n i}^{(\nu)}\right|<k^{\nu}(n+1)^{\nu-1} n^{\nu \lambda n}, \\
& \quad n=1,2,3, \cdots ; i=0,1,2, \cdots, n .
\end{aligned}
$$

By (3.3) and (3.6)

$$
\begin{aligned}
\left|\pi_{n i}\right| & \leqq \sum_{\nu=1}^{m-1} m_{c_{\nu+1}}\left|p_{n i}^{(\nu)}\right| \quad(i=0,1,2, \cdots, n-1) \\
& <\sum_{\nu=1}^{m-1} m_{c_{\nu+1}} k^{\nu}(n+1)^{\nu-1} n^{\nu \lambda n}
\end{aligned}
$$

that is,

$$
\left|\pi_{n i}\right|<2^{m} k^{m-1}(n+1)^{m-2} n^{(m-1) \lambda n} .
$$

Also

$$
\begin{aligned}
A_{i}(R) & \leqq \sum_{j=0}^{i}\left|p_{i j}\right| R^{i} & \\
& <k(i+1) R^{i} i^{\lambda i} & (R>1 ; i=1,2,3, \cdots) \\
& <k(n+1) R^{n} n^{\lambda n} & (i=0,1,2, \cdots, n)
\end{aligned}
$$


so that

$$
\omega_{n}(R)<2^{m} k^{m}(n+1)^{m} R^{n} n^{m \lambda n}, \quad n=1,2,3, \cdots,
$$

from which $\omega \leqq m \lambda$. Thus the set $\left\{p_{n}(z)\right\}$ is of order at most $m \lambda$. We next write $\left\{p_{n}(z)\right\}^{v}=\left\{p_{n}^{(\nu)}(z)\right\}, \Pi^{\nu}=\left[\pi_{n i}^{(\nu)}\right]$,

$$
\omega_{n}^{(\nu)}(R)=\sum_{i=0}^{n}\left|\pi_{n i}^{(v)}\right| A_{i}^{(\nu)}(R), \quad A_{i}^{(\nu)}(R)=\max _{|z|=R}\left|p_{i}^{(\nu)}(z)\right| .
$$

Multiplying (3.2) by $\Pi^{2}=P^{-2}$ we get

$$
\Pi^{2}=m_{c_{1}} \Pi-m_{c_{2}} I+m_{c_{3}} P-\cdots+(-1)^{m-1} P^{m-2},
$$

from which

$$
\pi_{n i}^{(2)}=m_{c_{1}} \pi_{n i}+\sum_{\nu=1}^{m-2}(-1)^{\nu+1} m_{c_{\nu+2}} p_{n i}^{(p)}, \quad i=0,1,2, \cdots, n-1,
$$

so that by (3.6) and (3.7),

$$
\left|\pi_{n i}^{(2)}\right|<m_{c_{1}} 2^{m} k^{m-1}(n+1)^{m-2} n^{(m-1) \lambda n}+\sum_{\nu=1}^{m-2} m_{c_{\nu+2}} k^{\nu}(n+1)^{\nu-1} n^{\nu \lambda n}
$$

or

$$
\left|\pi_{n i}^{(2)}\right|<2^{2 m} k^{m-1}(n+1)^{m-2} n^{(m-1) \lambda n}
$$

Also,

$$
\begin{aligned}
A_{i}^{(2)}(R) & \leqq \sum_{j=0}^{i}\left|p_{i j}^{(2)}\right| R^{j} \\
& <\sum_{j=0}^{i} k^{2}(i+1) i^{2 \lambda i} R^{j} \\
& <k^{2}(n+1)^{2} R^{n} n^{2 \lambda n}, \quad i=0,1,2, \cdots, n .
\end{aligned}
$$

Whence

$$
\omega_{n}^{(2)}(R)<2^{2 m} k^{m+1}(n+1)^{m+1} R^{n} n^{(m+1) \lambda n},
$$

so that $\omega^{(2)} \leqq(m+1) \lambda$.

Again, multiplying (3.2) by $\Pi^{3}=P^{-3}$ we get

$$
\Pi^{3}=m_{c_{1}} \Pi^{2}-m_{c_{2}} \Pi+m_{c_{3}} I-m_{c_{4}} P+\cdots+(-1)^{m-1} P^{m-8} .
$$

Whence by (3.6), (3.7), and (3.8)

$$
\left|\pi_{n i}^{(3)}\right|<2^{3 m} k^{m-1}(n+1)^{m-2} n^{(m-1) \lambda n} .
$$


It appears clear that for any $\nu$,

$$
\left|\pi_{n i}^{(\nu)}\right|<2^{\nu m} k^{m-1}(n+1)^{m-2} n^{(m-1) \lambda n} .
$$

Also

$$
\begin{aligned}
A_{i}^{(8)}(R) & \leqq \sum_{j=0}^{2}\left|p_{i j}^{(3)}\right| R^{j} \\
& <k^{3}(n+1)^{3} R^{n} n^{3 \lambda n}
\end{aligned}
$$

which leads with (3.9) to

$$
\omega^{(3)} \leqq(m+2) \lambda .
$$

In general for any $\nu$,

$$
A_{i}^{(\nu)}(R)<k^{\nu}(n+1)^{p} R^{n} n^{\nu \lambda n}
$$

which gives with (3.10),

$$
\omega^{(\nu)} \leqq(m+\nu-1) \lambda .
$$

As a special case

$$
\omega^{(m-1)} \leqq 2(m-1) \lambda .
$$

Now from (2.1)

$$
P^{m}=m_{c_{1}} P^{m-1}-m_{c_{2}} P^{m-2}+\cdots+(-1)^{m-1} I,
$$

so that

$$
\begin{aligned}
\left|p_{n i}^{(m)}\right| & \leqq \sum_{\nu=1}^{m-1} m_{c_{\nu}}\left|p_{n i}^{(v)}\right| \quad(i=0,1,2, \cdots, n-1) \\
& <2^{m} k^{m-1}(n+1)^{m-2} n^{(m-1) \lambda n} \quad(\text { by } 3.6) .
\end{aligned}
$$

This gives with (3.10)

$$
\omega^{(m)} \leqq 2(m-1) \lambda .
$$

In the same way, the relation

$$
P^{m+\nu}=m_{c_{1}} P^{m+\nu-1}-m_{c_{2}} P^{m+\nu-2}+\cdots+(-1)^{m-1} P^{\nu}
$$

leads to

$$
\omega^{(m+\nu)} \leqq 2(m-1) \lambda, \quad \text { all } \nu \geqq 1 .
$$

This completes the proof of the theorem.

The fact that the upper bound may be attained will be illustrated in $\$ 5$ by means of an example. 
4. The simple monic set in which the operators $\pi_{n i}$ satisfy either $\left|\pi_{n i}\right| \leqq k n^{\lambda}$ or $\left|\pi_{n i}\right| \leqq k n^{\lambda(n-i)}, n=1,2,3, \cdots, i=0,1,2, \cdots, n-1$, has been studied $[6 ; 2]$; the order in either case may attain its upper bound $\lambda$. We consider here the case in which $\left|\pi_{n i}\right| \leqq k n^{\lambda n}$ and the set is algebraic.

Multiplying both sides of $(2.1)$ by $\Pi^{m}=P^{-m}$, we get

$$
I-m_{c_{1}} \Pi+m_{c_{2}} \Pi^{2}-\cdots+(-1)^{m} \Pi^{m}=0,
$$

so that the statement that the simple monic set $\left\{p_{n}(z)\right\}$ is algebraic of degree $m$ means both equations $(P-I)^{m}=0$ and $(\Pi-I)^{m}=0 .{ }^{3}$

By a treatment similar to that in Theorem 1 and using the equation

$$
P=m_{c_{1}} I-m_{c_{2}} \Pi+m_{c_{3}} \Pi^{2}-\cdots+(-1)^{m-1} \Pi^{m-1}
$$

obtained from (4.1) to determine the coefficients $p_{i j}$, we obtain the following results.

ThEOREM 2. If $\left\{p_{n}(z)\right\}$ is an algebraic simple monic set of degree $m$ in which

$$
\left|\pi_{n i}\right| \leqq k n^{\lambda n}, k \geqq 1, n=1,2,3, \cdots ; i=0,1,2, \cdots, n-1,
$$

then

$\left\{p_{n}(z)\right\}^{\nu}$ is of order at most $(m+\nu-1) \lambda, 1 \leqq \nu \leqq m-1$,

$\left\{p_{n}(z)\right\}^{\nu}$ is of order at most $2(m-1) \lambda, \nu \geqq m-1$.

The upper bound may be attained in all cases.

5. The construction of an example to show that $\left\{p_{n}(z)\right\}$ ' may actually be of order $(m+\nu-1) \lambda$, for a given value of $m$ and any assigned value of $\nu, 1 \leqq \nu \leqq m-1$, may seem to be of some difficulty. We give here one and the same example for the values $m=3$ and $\nu=1, \nu=2$. A detailed study of the example will make it clear that we can actually construct an example for any value of $m$ and any value of $\nu$, provided that we have enough time and plenty of paper.

Consider the set $\left\{p_{n}(z)\right\}$ defined by:

$$
\begin{aligned}
& p_{6 h}(z)=z^{6 h} \\
& p_{6 h+1}(z)=4 \mu(h) z^{6 h}+z^{6 h+1} \\
& p_{6 h+2}(z)=\quad 9 \mu(h) z^{6 h+1}+z^{6 h+2} \\
& p_{6 h+8}(z)=\quad 3 \mu(h) z^{6 h+1}+z^{6 h+3} \\
& p_{6 h+1}(z)=\quad-2 \mu(h) z^{6 h+2}+6 \mu(h) z^{6 h+3}+z^{6 h+4} \\
& p_{6 h+5}(z)= \\
& 6 \mu(h) z^{6 h+4}+z^{6 h+5},
\end{aligned}
$$

${ }^{3}$ It follows also that if $\left\{p_{n}(z)\right\}$ is an algebraic simple monic set then its reciprocal set $\left\{\bar{p}_{n}(\mathbf{s})\right\}[3]$ satisfies the same algebraic equation. 
The reduced characteristic equation of the square matrix

$$
A=\left[\begin{array}{cccccc}
1 & 0 & 0 & 0 & 0 & 0 \\
4 \mu & 1 & 0 & 0 & 0 & 0 \\
0 & 9 \mu & 1 & 0 & 0 & 0 \\
0 & 3 \mu & 0 & 1 & 0 & 0 \\
0 & 0 & -2 \mu & 6 \mu & 1 & 0 \\
0 & 0 & 0 & 0 & 6 \mu & 1
\end{array}\right]
$$

is easily verified to be $(A-I)^{3}=0$. Since the matrix $P$ of coefficients of the set $\left\{p_{n}(z)\right\}$ consists of blocks of the type $A$, the method of partitionization gives the corresponding equation $(P-I)^{3}=0$, so that the set $\left\{p_{n}(z)\right\}$ is algebraic of degree 3 .

(An algebraic semi-lower matrix does not need to consist of such blocks, for example, the infinite matrix

$$
\left[\begin{array}{lllllll}
1 & & & & & & \\
p_{10} & 1 & & & & \\
0 & 0 & 1 & & & \\
p_{30} & 0 & p_{32} & 1 & & \\
0 & 0 & 0 & 0 & 1 & \\
p_{50} & 0 & p_{52} & 0 & p_{54} & 1 \\
. & . & . & . & . & . & .
\end{array}\right]
$$

is algebraic of degree 2. But every semi-lower matrix which consists of such blocks of $s$ rows and $s$ columns is algebraic of degree $s$ at most.)

From the relation $A^{3}-3 A^{2}+3 A-I=0$ we have

$$
A^{-1}=3 I-3 A+A^{2} \text {. }
$$

It can be easily verified that

$$
A^{2}=\left[\begin{array}{cccccc}
1 & 0 & 0 & 0 & 0 & 0 \\
8 \mu & 1 & 0 & 0 & 0 & 0 \\
36 \mu^{2} & 18 \mu & 1 & 0 & 0 & 0 \\
12 \mu^{2} & 6 \mu & 0 & 1 & 0 & 0 \\
0 & 0 & -4 \mu & 12 \mu & 1 & 0 \\
0 & 0 & -12 \mu^{2} & 36 \mu^{2} & 12 \mu & 1
\end{array}\right]
$$

and 


$$
A^{-1}=\left[\begin{array}{cccccc}
1 & 0 & 0 & 0 & 0 & 0 \\
-4 \mu & 1 & 0 & 0 & 0 & 0 \\
36 \mu^{2} & -9 \mu & 1 & 0 & 0 & 0 \\
12 \mu^{2} & -3 \mu & 0 & 1 & 0 & 0 \\
0 & 0 & 2 \mu & -6 \mu & 1 & 0 \\
0 & 0 & -12 \mu^{2} & 36 \mu^{2} & -6 \mu & 1
\end{array}\right]
$$

so that

$$
\begin{aligned}
z^{6 h+5}= & -12 \mu^{2}(h) p_{6 h+2}(z)+36 \mu^{2}(h) p_{6 h+3}(z) \\
& -6 \mu(h) p_{6 h+4}(z)+p_{6 h+5}(z) \\
\omega_{6 h+5}(R)> & 36 \mu^{2}(h) \cdot A_{6 h+3}(R) \\
> & 36 \mu^{2}(h) \cdot 3 \mu(h) R^{6 h+1}
\end{aligned}
$$

Therefore

$$
\lim _{h \rightarrow \infty} \frac{\log \omega_{6 h+5}(R)}{(6 h+5) \log (6 h+5)} \geqq 3 \lambda .
$$

By Theorem 1, the set $\left\{p_{n}(z)\right\}$ is of order $3 \lambda$.

The reciprocal set, $\left\{p_{n}(z)\right\}$, of the above set is one satisfying (4.2). For this set we have

$$
\begin{aligned}
z^{6 h+4} & =-2 \mu(h) \bar{p}_{6 h+2}(z)+6 \mu(h) \bar{p}_{6 h+3}(z)+\bar{p}_{6 h+4}(z), \\
\bar{\omega}_{6 h+4}(R) & >2 \mu(h) \cdot \bar{A}_{6 h+2}(R) \\
& >2 \mu(h) \cdot 36 \mu^{2}(h) R^{6 h},
\end{aligned}
$$

so that $\bar{\omega}=3 \lambda$, by Theorem 2 .

It can also be verified that

$$
A^{-2}=3 A^{-1}-3 I+\dot{A}=\left[\begin{array}{cccccc}
1 & 0 & 0 & 0 & 0 & 0 \\
-8 \mu & 1 & 0 & 0 & 0 & 0 \\
108 \mu^{2} & -18 \mu & 1 & 0 & 0 & 0 \\
36 \mu^{2} & -6 \mu & 0 & 1 & 0 & 0 \\
0 & 0 & 4 \mu-12 \mu & 1 & 0 \\
0 & 0 & -36 \mu^{2} & 108 \mu^{2}-12 \mu & 1
\end{array}\right] \text {, }
$$

so that

$$
\begin{aligned}
z^{6 h+5}= & -36 \mu^{2}(h) p_{6 h+2}^{(2)}(z)+108 \mu^{2}(h) p_{6 h+3}^{(2)}(z)-12 \mu(h) p_{6 h+4}^{(2)}(z) \\
& +p_{6 h+5}^{(2)}(z),
\end{aligned}
$$




$$
\begin{aligned}
\omega_{6 h+5}^{(2)}(R) & >36 \mu^{2}(h) \cdot A_{6 h+2}^{(2)}(R) \\
& >36 \mu^{2}(h) \cdot 36 \mu^{2}(h) R^{6 h},
\end{aligned}
$$

so that $\omega^{(2)}=4 \lambda$, by Theorem 1 .

For the reciprocal set $\left\{p_{n}(z)\right\}$ we have

$$
\begin{aligned}
z^{6 h+5}= & -12 \mu^{2}(h) \bar{p}_{6 h+2}^{(2)}(z)+36 \mu^{2}(h) \bar{p}_{6 h+3}^{(2)}(z)+12 \mu(h) \bar{p}_{6 h+4}^{(2)}(z) \\
& +\bar{p}_{6 h+5}^{(2)}(z), \\
\bar{\omega}_{6 h+5}^{(2)}(R)> & 36 \mu^{2}(h) \cdot \bar{A}_{6 h+3}^{(2)}(R) \\
> & 36 \mu^{2}(h) \cdot 36 \mu^{2}(h) R^{6 h},
\end{aligned}
$$

so that $\bar{\omega}^{(2)}=4 \lambda$, by Theorem 2 .

6. If $\left\{p_{n}(z)\right\}$ is a simple set in which the zeros of $p_{n}(z)$ all lie in $|z| \leqq k n^{\lambda}$ then [4] $\left\{p_{n}(z)\right\}$ represents every integral function of order less than $1 /(\lambda+1)$ but may not represent an integral function of order $1 /(\lambda+1)$. When we consider algebraic sets we get the following result.

THEOREM 3. If $\left\{p_{n}(z)\right\}$ is an algebraic simple monic set in which the zeros of $p_{n}(z)$ all lie in the circle $|z| \leqq k n^{\lambda}$, then $\left\{p_{n}(z)\right\}$ represents every integral function of order less than $1 / \lambda$.

We have

therefore

$$
p_{n}(z)=\left(z-a_{n 1}\right)\left(z-a_{n 2}\right) \cdots\left(z-a_{n n}\right)=\sum_{i=0}^{n} p_{n i} z^{i},
$$

$$
\left|p_{n i}\right| \leqq n_{c_{i}} k^{n-i} n^{\lambda(n-i)}<2^{n} k^{n-i} n^{\lambda(n-i)} .
$$

Proceeding as in Theorem 1,

$$
\left|p_{n i}^{(2)}\right|<\sum_{j=i}^{n} 2^{n} k^{n-j} n^{\lambda(n-j)} \times 2^{j} k^{j-i} i^{\lambda(j-i)}<2^{2 n} k^{n-i}(n+1) n^{\lambda(n-i)} .
$$

In general

$$
\left|p_{n i}^{(\nu)}\right|<2^{\nu n} k^{n-i}(n+1)^{n-1} n^{\lambda(n-i)},
$$

therefore

$$
\left|\pi_{n i}\right| \leqq \sum_{\nu=1}^{m-1} m_{c_{\nu+1}}\left|p_{n i}^{(\nu)}\right|<2^{m} \cdot 2^{(m-1) n} k^{n-i}(n+1)^{m-2} n^{\lambda(n-i)} .
$$

Also 


$$
A_{i}(R)<\sum_{j=0}^{i} 2^{i} k^{i-i i^{\lambda(i-j)}} R^{i}<2^{i} k^{i} R^{i}(i+1) \cdot i^{\lambda i}, \quad R>1 .
$$

Therefore

$$
\omega_{n}(R)<2^{(m+1) n} k^{n} R^{n}(n+1)^{m-1} n^{\lambda n} .
$$

Therefore the set is of order at most $\lambda$, and hence the result.

7. If $\left\{p_{n}(z)\right\}$ and $\left\{q_{n}(z)\right\}$ are two simple monic sets, then the sum set $\left\{u_{n}(z)\right\}=\left[\alpha\left\{p_{n}(z)\right\}+\beta\left\{q_{n}(z)\right\}\right] /(\alpha+\beta), \alpha+\beta \neq 0$, is also a simple monic set. It is quite remarkable that the two sets $\left\{p_{n}(z)\right\}$ and $\left\{q_{n}(z)\right\}$ may be of finite order, $\lambda$ say, and the set $\left\{u_{n}(z)\right\}$ is of infinite order. Thus if

$$
p_{0}(z)=1, \quad p_{n}(z)=2 \cdot n^{\lambda} z^{n-1}+z^{n}, \quad n \geqq 1,
$$

and

$q_{0}(z)=1, \quad q_{n}(z)=z^{n}, n$ odd, $\quad q_{n}(z)=\left(-2 \cdot n^{\lambda}+2 \cdot n^{n \lambda}\right) z^{n-1}+z^{n}, \quad n$ even, then $\left\{p_{n}(z)\right\}$ and $\left\{q_{n}(z)\right\}$ are both of order $\lambda$, while $\left\{u_{n}(z)\right\}=\left[\left\{p_{n}(z)\right\}\right.$ $\left.+\left\{q_{n}(z)\right\}\right] / 2$, defined by,

$u_{0}(z)=1, \quad u_{n}(z)=n^{\lambda} z^{n-1}+z^{n}, n$ odd, $\quad u_{n}(z)=n^{n \lambda} z^{n-1}+z^{n}, \quad n$ even, is of infinite order.

It is clear that the set $\left\{u_{n}(z)\right\}$ is not algebraic. When we assume that the sum set $\left\{u_{n}(z)\right\}$ is algebraic, we obtain the following interesting result.

THEOREM 4. If $\left\{p_{n}^{\nu}(z)\right\}, \nu=1,2,3, \cdots, s$, are simple monic sets of order $\lambda$ each, then the sum set

$$
\begin{aligned}
\left\{u_{n}(z)\right\} & =\frac{1}{\alpha_{1}+\alpha_{2}+\cdots+\alpha_{s}}\left[\alpha_{1}\left\{p_{n}^{1}(z)\right\}+\cdots+\alpha_{s}\left\{p_{n}^{8}(z)\right\}\right], \\
\sum \alpha_{\nu} & \neq 0
\end{aligned}
$$

if algebraic of degree $m$, is of order at most $m \lambda$.

For any fixed value of $R$, say $R>1$,

$$
\omega_{n}^{\prime \prime}(R)<k_{\nu} n^{\lambda^{\prime} n}, \quad n \geqq 0, \lambda^{\prime}>\lambda,
$$

therefore

$$
\left|p_{n i}^{\prime}\right| R^{i} \leqq A_{n}^{\prime}(R) \leqq \omega_{n}^{\prime}(R)<k_{n} n^{\lambda^{\prime} n}
$$

therefore 


$$
\left|p_{n i}^{\prime}\right|<k_{r} n^{\lambda^{\prime} n}, \quad \quad k_{r}=\max \left(k_{1}, k_{2}, \cdots, k_{s}\right),
$$

therefore

$$
\left|u_{n i}\right|<k n^{\lambda^{\prime} n}, \quad \quad k=k_{r} \cdot \sum\left|\alpha_{\nu}\right| /\left|\sum \alpha_{\nu}\right| .
$$

Since $\left\{u_{n}(z)\right\}$ is an algebraic simple monic set, then, by Theorem 1, it is of order at most $m \lambda^{\prime}$. Since $\lambda^{\prime}$ is arbitrary, greater than $\lambda,\left\{u_{n}(z)\right\}$ is of order at most $m \lambda$.

The fact that the order $m \lambda$ may be attained is illustrated by the following example:

$$
\begin{aligned}
p_{6 h+1}(z) & =8 \mu(h) z^{6 h}+z^{6 h+1}, \\
b_{6 h+4}(z) & =-4 \mu(h) z^{6 h+2}+12 \mu(h) z^{6 h+3}+z^{6 h+4}, \\
p_{n}(z) & =z^{n}, \quad n=6 h, 6 h+2,6 h+3,6 h+5, \\
++2 & =18 \mu(h) z^{6 h+1}+z^{6 h+2}, \\
q_{6 h+3}(z) & =6 \mu(h) z^{6 h+1}+z^{6 h+3}, \\
q_{6 h+5}(z) & =12 \mu(h) z^{6 h+4}+z^{6 h+5}, \quad n=6 h, 6 h+1,6 h+4, \\
q_{n}(z) & =z^{n}, \quad n
\end{aligned}
$$

where

$$
\mu(h)=(6 h+1)^{\lambda(6 h+1)}, \quad h \geqq 0 .
$$

Each of the sets $\left\{p_{n}(z)\right\}$ and $\left\{q_{n}(z)\right\}$ is of order $\lambda$. The sum set $\left\{u_{n}(z)\right\}=\left[\left\{p_{n}(z)\right\}+\left\{q_{n}(z)\right\}\right] / 2$ is the set given in $\S 5$. Thus the sum set is algebraic of degree 3 and of order $3 \lambda$.

\section{REFERENCES}

1. Raouf $\mathrm{H}$. Makar, The infinite matrix series $\sum A^{r}$ and the field $P_{\mathbf{A}}$, Proceedings of the Mathematical and Physical Society of Egypt vol. 3 (1948).

2. M. Mursi and Ragy H. Makar, Coefficients of basic sets and functions represented, Proceedings of the Mathematical and Physical Society of Egypt vol. 3 (1945).

3. - Sur la base inverse d'une base de polynomes, Bull. Sci. Math. (2) vol. 71 (1947).

4. M. Nassif, On the zeros of basic sets of polynomials, Proceedings of the Mathematical and Physical Society of Egygt vol. 2 (1944).

5. H. W. Turnbull and A. C. Aitken, The theory of canonical matrices, London and Glasgow, 1932.

6. J. M. Whittaker, On series of polynomials, Quart. J. Math. Oxford Ser. vol. 5 (1934).

7. _- Sur les series de base de polynomes quelconques, Paris, 1949.

FOUAD I UNIVERSITY 